EPJ Web of Conferences 59, 16002 (2013)

DOI: $10.1051 /$ epjconf/20135916002

(C) Owned by the authors, published by EDP Sciences, 2013

\title{
Testing ion structure models with x-ray Thomson scattering
}

\author{
K. Wünsch, J. Vorberger and D.O. Gericke
} Centre for Fusion, Space and Astrophysics, Department of Physics, University of Warwick,
Coventry CV4 7AL, UK

\begin{abstract}
We investigate the influence of various ionic structure models on the interpretation of the X-ray Thomson scattering signal. For the calculation of the ion structure, classical hypernetted chain equations are used applying different effective inter-particle potentials. It is shown that the different models lead to significant discrepancies in the theoretically predicted weight of the Rayleigh peak, in particular for small $k$-values where correlation effects are important. Here, we propose conditions which might allow for an experimental verification of the theories under consideration of experimental constraints of $k$-vector blurring.
\end{abstract}

\section{INTRODUCTION}

The availability of high-energy laser facilities, in particular the National Ignition Facility (NIF) at the Lawrence Livermore National Laboratory, USA, makes it nowadays possible to create highly compressed matter with densities exceeding solids and moderate to high temperatures [1]. Materials under these conditions possess properties which are common to solids, fluids and plasmas [2]. This combination makes the theoretical investigation challenging as strongly coupled ions and degenerate electrons have to be treated simultaneously. A better understanding of these extreme states of matter, often referred to as warm dense matter (WDM), is of high interest for the modeling of ICF experiments where such states occur in the Hohlraum walls and, as a transient state, in the fusion capsule during the compression phase [3].

Beside the generation, the diagnostics of WDM poses a challenge as the high particle densities prohibit the application of standard plasma techniques such as optical spectroscopy. X-ray Thomson scattering (XRTS) avoids these problems and has been established as a diagnostic tool capable of delivering basic plasma parameters, such as electron density, mass density, temperature and ionization degree, of the material under investigation [4]. The performance of currently available x-ray sources, that is, the bandwidth and the time duration of the probe beam, also allows the study of the structural properties of the target material as well as the dynamic response of the system [5-7]. Hitherto the experimental constraints of current laser-based x-ray sources only allow the access of the electron motions, as the ion acoustic modes have frequencies lower than the ion plasma frequency. However, this low-frequency part might be accessible in near future with the availability of $\mathrm{x}$-ray free electron lasers, for instance at LCLS [8].

The scattering power per solid angle $\mathrm{d} \Omega$ and per frequency $\mathrm{d} \omega$ is directly proportional to the microscopic structure of the electrons characterised by the electron dynamic structure factor $S_{e e}^{\text {tot }}(\mathbf{k}, \omega)$ [9]

$$
\frac{P_{s}(\mathbf{k}, \omega)}{\mathrm{d} \omega \mathrm{d} \Omega} \propto S_{e e}^{\mathrm{tot}}(\mathbf{k}, \omega)
$$

This is an Open Access article distributed under the terms of the Creative Commons Attribution License 2.0, which permits unrestricted use, distribution, and reproduction in any medium, provided the original work is properly cited. 
where $\mathbf{k}$ and $\omega$ are the wavenumber and the frequency shift of the incident photons during the scattering process, respectively. The dynamic structure factor, describing the microscopic density fluctuations in the systems, plays thus a crucial role when investigating light scattering theoretically.

The scattering spectra possess two distinct features: (i) the unshifted Rayleigh peak and (ii) a frequency-shifted feature. Following the idea of Chihara, the dynamic structure factor in partially ionised, multicomponent plasmas can be decomposed with respect to the different scattering features (the wave number in the arguments are suppressed for simplicity) [10-12]

$S_{e e}^{\mathrm{tot}}(\omega)=\sum_{a, b} \sqrt{x_{a} x_{b}}\left[f_{a}+q_{a}\right]\left[f_{b}+q_{b}\right] S_{a b}(\omega)+\bar{Z} S_{e e}^{0}(\omega)+\sum_{a} Z_{a}^{c} x_{a} \int d \omega^{\prime} \tilde{S}_{a}^{c e}\left(\omega-\omega^{\prime}\right) S_{a}^{S}\left(\omega^{\prime}\right)$.

Here, $a$ and $b$ run over all ion species, $x_{a}=n_{a} / \sum_{a} n_{a}$ denote the concentrations of the species given by ion densities $n_{a}$, and $Z_{a}$ is the charge of the ions of species $a$. The first term describes the unshifted Rayleigh peak due to elastic scattering on electrons co-moving with the ions, that is, bound electrons described by the ion form factor $f_{a}(k)$ and the electrons forming the screening cloud accounted for by the function $\left.q_{a}(k) . S_{a b} k, \omega\right)$ reflects the spatial arrangements and the thermal motion of the ions. As already pointed out, this low frequency part cannot be resolved with current x-ray sources which allows for a static treatment, that is, $S_{a b}(k, \omega)=S_{a b}(k) \delta(\omega)$. The second term of Eq. (2) describes inelastic scattering from kinetically free electrons. As these electrons are often only weakly coupled, their contribution can be described by perturbation theory, e.g., the random phase approximation. The last term in Eq. (2) accounts for the inelastic scattering caused by excitations of the inner core electrons.

In this article, we concentrate on the elastic scattering feature that contains information on the ionic subsystem, such as ion density, ion temperature and charge state. To extract these parameters from the scattering signal, an excellent theoretical description of all quantities involved is required, as the theoretically generated spectrum is fitted to the experimental measured profile. Such a theory is a particular challenging task, especially as the effective force between the ions is not known in WDM. Strong forces and electron screening produce a nontrivial ion structure which is particularly uncertain in the the small wave number regime. Here, several models for the ion-ion forces and, thus, the ion-ion structure factor, will be discussed. It is then investigated whether experimental XRTS signals can be used to distinguish between the models and what the requirements for such measurements would be. Finally, the influence of the different models for the ion structure on the interpretation of the measured XRTS signal will be studied.

\section{ION STRUCTURE MODELS IN HIGH-ENERGY-DENSITY MATTER}

For the investigation of the ion structure, full quantum simulations, i.e., density functional molecular dynamics (DFT-MD), and classical hypernetted chain equations (HNC) are applied. Whereas DFT-MD fully meets the requirements of WDM, i.e, the consistent description of strong inter-particle forces and degenerate electrons, the high computational demand limits its applicability as an effective analysis tool. In contrast, the HNC approach is numerically very efficient. This method was however developed for classical systems where it yields reasonably accurate results even for strongly coupled systems [13]. To apply this methods in the WDM regime too, adjustments to the inter-particle potential, which is an input quantity here, have to be made to consider screening by bound electrons.

Here, three models in the HNC approach will be considered: (i) the one-component plasma (OCP) model; (ii) the Yukawa model (Y); and (iii) the Yukawa model with a short range repulsion term $(\mathrm{Y}+\mathrm{SRR})$. The OCP model considers the ions to interact via bare Coulomb forces and the electrons as a uniform and structureless background added to ensure quasi-neutrality of the system. In the Yukawa model, the electrons are treated as a polarizable background resulting in a statically screened ion-ion 

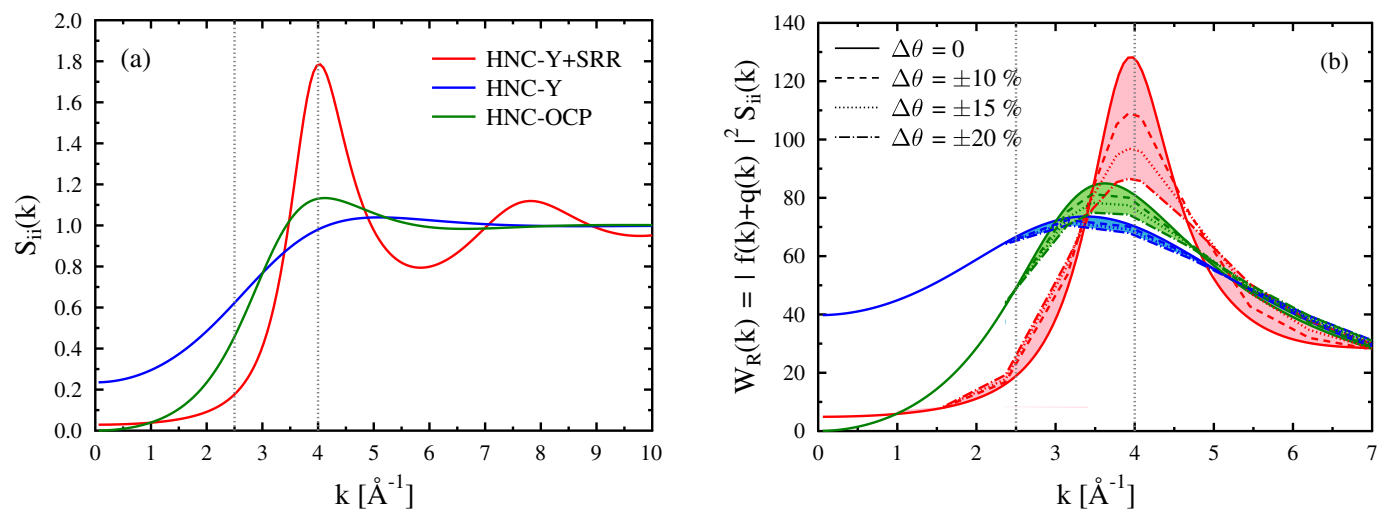

Figure 1. a) Static structure factor and (b) weight of the Rayleigh peak for an aluminium plasma with $T=10 \mathrm{eV}$, $3 \times$ solid density $\left(n_{i}=1.8 \times 10^{23} \mathrm{~cm}^{-3}\right)$ and $Z=3$ calculated for three different potentials with the HNC approach: unscreened OCP, screened Yukawa $(\mathrm{Y})$ and the Yukawa plus a short-range repulsion $(\mathrm{Y}+\mathrm{SRR}) . W_{R}(k)$ includes $k$-vector blurring to account for uncertainties in the scattering angle $\theta$. The grey dashed lines indicate two wave vectors where the largest discrepancies between the models can be observed.

potential of the form [14]

$$
V_{i i}^{\mathrm{Y}}(r)=\frac{Z_{i}^{2} e^{2}}{r} \exp \left(-\kappa_{s} r\right) \quad \text { with } \quad \kappa_{s}^{2}=\frac{4 e^{2} m_{e}}{\pi \hbar^{3}} \int_{0}^{\infty} \mathrm{d} p f_{e}(p) .
$$

Here, $\kappa_{s}$ denotes the inverse of the electron screening length which may be calculated from the Fermi distribution $f_{e}(p)$ of the electrons to account for partial degeneracy in WDM. The last model includes an additional short-range repulsion term added to the screened Coulomb potential [15]

$$
V^{Y+S R R}(r)=\left(\frac{a}{r}\right)^{4}+\frac{Z^{2} e^{2}}{r} \exp (-\kappa r) .
$$

The latter term accounts for bound electrons in partially ionized systems. This expression was obtained via direct comparisons of the structure factors calculated by DFT-MD and HNC [15]. The parameter $a$ is here a fit parameter to match the HNC results to the DFT-MD data.

Figure 1 shows the static structure factor for warm dense aluminium obtained by the HNC approach applying the models described above. The plasma parameters are $T=10 \mathrm{eV}$, three-fold solid density and $Z=3$ yielding a strongly coupled plasma with a classical coupling parameter of $\Gamma=15$ for the ions. The HNC calculations applying the OCP model give a structure factor typical for moderately coupled systems, that is, a correlation hole for small wave numbers and the rise of a maximum around $k=4 \AA^{-1}$. As screening effects are neglected in this model, $S_{i i}(k)$ approaches zero at the origin. The use of a screened Coulomb potential (Yukawa model) yields an increase of the structure factor for small wave vectors $k$ and a reduced maximum at intermediate $k$. As aluminium is only partially ionized, the forbidden overlap of the electron orbitals of the bound electrons cause an additional repulsion force for small distances. Including this effect by applying the Y+SRR potential in the HNC method leads to significant changes in the structural behaviour: the correlation hole increases and well-pronounced oscillations appear which indicate the formation of a short-range structure in the ion fluid. 


\section{INFLUENCE OF THE ION STRUCTURE MODELS ON THE ELASTIC RAYLEIGH PEAK}

In most experiments, the measured elastic scattering is fully characterised by the frequency integrated area under the Rayleigh peak. This quantity is also referred to as the weight of the Rayleigh peak

$$
W_{R}(k)=\left[f_{i}(k)+q(k)\right]^{2} S_{i i}(k) .
$$

For the evaluation of this quantity, the structure factor, the form factor and the screening function are required. The screening function is calculated here in linear response to a Coulomb field, that is, $q(k)=Z \kappa_{s}^{2} / \kappa_{s}^{2}+k^{2}$ [16]. The form factor is calculated for an isolated ions via DFT.

The solid lines in Fig. 1(b) present the weight of the Rayleigh peak for an aluminium plasma using the static structure factors shown in the left panel, Fig. 1(a). It can be seen that the various models for the ion structure strongly affect the predictions of the scattering signal. For an experimental verification, the wave vectors probed should be chosen at the position where the most discrepancies arise as indicated by the grey dashed lines.

For direct comparisons with measured data, uncertainties in the scattering angle arising due to the finite sample size and scattering geometry have to be taken into account. To account for this effect, the theoretical results are convolved with a Gaussian instrumental response function to account for $k$-vector blurring. The results are presented in Fig. 1(b) where errors up to $\pm 20 \%$ (of $\Delta \theta=40 \%$ ) are considered. $k$-vector blurring has only a significant effect around the peak position. The reason for this behaviour lies in the characteristics of $W_{R}(k)$, which is strongly nonlinear around the peak position and approximately linear for small $k$-values. Therefore, the peak in $W_{R}(k)$ is significantly reduced by increasing the uncertainty of the probe wave vector. This fact might complicate the experimental distinction between the theoretical models as currently available measured scattering profiles still possess experimental uncertainties in the order of $20 \%$ [4].

\section{CONCLUSION}

The different models for the ion-ion interaction significantly change the static ion structure and, thus, the theoretically predicted strength of the elastic feature in the scattering spectrum. To apply a correct ion structure is particularly important in the collective scattering regime, as the height of the Rayleigh peak is used here to extract the temperature and the charge state of the system. Thus, an experimental verification of the various models is highly desirable for an advancement of x-ray Thomson scattering as a reliable diagnostic tool in WDM. For an optimal test, a strongly coupled, partially ionized system should be considered as the different theories predict here the larges differences. Furthermore, the wave vector probed should be chosen carefully to access the region with the largest discrepancies under the experimental constraints.

This work was supported by the Engineering and Physical Sciences Research Council and via an Early Career Fellowship from the Institute of Advanced Studies (University of Warwick, UK) for KW.

\section{References}

[1] S.H. Glenzer et al., Science 327, 1228 (2010)

[2] National Research Council, Frontier in High Energy Density Physics (The National Academy Press, Washington, 2003)

[3] S. Atzeni and J. Meyer-ter-Vehn, The Physics of Inertial Fusion (Clarendon Press, Oxford, 2004) 


\section{IFSA 2011}

[4] S. H. Glenzer and R. Redmer, Rev. Mod. Phys. 81, 1625 (2009)

[5] M. K. Urry et al., J. Quant. Spectrosc. Radiat. Transfer 99, 636 (2006)

[6] E. Garcia Saiz et al., Nature Phys. 4, 940 (2008)

[7] A. Pelka et al., Phys. Rev. Lett. 105, 265701 (2010)

[8] G. Gregori and D. O. Gericke, Phys. Plasmas 16, 056306 (2009)

[9] I. H. Hutchinson, Principles of Plasma Diagnostics (Cambridge University Press, 2005)

[10] J. Chihara, J. Phys. F: Met. Phys. 17, 295 (1987)

[11] J. Chihara, J. Phys.: Condens. Matter 12, 231 (2000)

[12] K. Wünsch, J. Vorberger, G. Gregori, and D. O. Gericke, EPL 94, 25001 (2011)

[13] K. Wünsch, P. Hilse, M. Schlanges, and D. O. Gericke, Phys. Rev. E 77, 056404 (2008)

[14] D. Kremp, M. Schlanges, and W.-D. Kraeft, Quantum Statistics of Nonideal Plasmas (Springer, Berlin, 2006)

[15] K. Wünsch, J. Vorberger, and D. O. Gericke, Phys. Rev. E 79, 010201(R) (2009)

[16] D. O. Gericke, K. Wünsch, J. Vorberger, and G. Gregori, Phys. Rev. E 81, 065401(R) (2010) 